\title{
Inhibitory effect of probiotic Lactobacillus supernatants from the oral cavity on Streptococcus mutans biofilms
}

\author{
Rodnei Dennis Rossoni*,1, Marisol dos Santos Velloso ${ }^{1}$, Patrícia Pimentel de Barros, \\ Janaina Araújo de Alvarenga, Jéssica Diane dos Santos, Ana Carolina Chipoletti dos Santos Prado, \\ Felipe de Camargo Ribeiro, Ana Lia Anbinder, Juliana Campos Junqueira \\ Department of Biosciences and Oral Diagnosis, São Paulo State University (Unesp), Institute of Science and Technology, São José dos Campos, São Paulo, Brazil
}

A R T I C L E I N F O

\section{Keywords:}

Streptococcus mutans

Lactobacillus

Supernatant

Biofilm

Oral pathogens

\begin{abstract}
A B S T R A C T
Probiotics can release bioactive substances that can inhibit the growth and biofilm formation of pathogenic microorganisms such as Streptococcus mutans. In this context, we evaluated whether the supernatants of Lactobacillus strains isolated from caries-free subjects can inhibit $S$. mutans, one of the most important bacteria for dental caries. First, the supernatants of 22 Lactobacillus strains were screened for antibacterial activity against S. mutans in planktonic cultures. All 22 Lactobacillus strains studied (100\%) showed antibacterial activity. Thereafter, the Lactobacillus strains with the greatest reductions in the planktonic $S$. mutans cultures were tested on biofilms. The L. fermentum 20.4, L. paracasei 11.6, L. paracasei 20.3 and L. paracasei 25.4 strains could significantly reduce the number of $S$. mutans cells in biofilms formed in hydroxyapatite $(p<0.05)$. This reduction was also confirmed by scanning electron microscopy analysis and was not caused by the decreased $\mathrm{pH}$ value in the medium $(p>0.05)$. In addition, the supernatants of these probiotic strains could also reduce the total biomass of $S$. mutans biofilms $(p<0.05)$. In conclusion, most of the Lactobacillus strains tested have some antibacterial activity against $S$. mutans. L. fermentum 20.4, L. paracasei 11.6 , L. paracasei 20.3 and L. paracasei 25.4 produce bioactive substances that caused a significant reduction in $S$. mutans biofilms.
\end{abstract}

\section{Introduction}

The term "probiotic" is used to describe live microorganisms that have beneficial effects on human health when administered in adequate amounts [1-4]. Some strains of the bacterial genera Lactobacillus and Bifidobacterium have been widely used as probiotics in several foods and dietary supplements to improve gastrointestinal health [5-7]. However, little is known about the effects of these strains on common oral infections, such as dental caries [8,9]. Previous studies suggested that consumption of dietary products containing probiotic lactobacilli reduces the number of Streptococcus mutans cells in saliva [8-10]. However, it is known that Lactobacillus spp. are acidogenic bacteria that can participate in the progression of dental caries [11]. Recently, it has been suggested that there are differences among various strains with respect to their ability to produce acid and that not all Lactobacillus strains have a caries-inducing effect [12].
Based on these observations, several authors have investigated the effects of certain Lactobacillus strains commonly used as probiotics in dietary products on the development of dental caries $[10,13,14]$. Söderling et al. [13] compared the effects of four probiotic Lactobacillus strains (Lactobacillus rhamnosus GG, Lactobacillus plantarum 229v, Lactobacillus reuteri SD2112 and L. reuteri PTA5289) on S. mutans biofilm formation. All of the Lactobacillus strains inhibited $S$. mutans biofilm formation on glass surfaces, although $L$. plantarum and $L$. reuteri PTA5289 showed a weaker inhibitory effect compared to L. reuteri SD2112 and $L$. rhamnosus. Marttinem et al. [14] also verified that $L$. reuteri ATCC PTA5289 could interfere with the adhesion of $S$. mutans to hydroxyapatite discs and inhibited biofilm formation. Lin et al. [10] studied other probiotic Lactobacillus strains, including Lactobacillus casei Shirota, Lactobacillus casei LC01, Lactobacillus plantarum ST-III, Lactobacillus paracasei Lpc-37 and Lactobacillus rhamnosus HN001. All five Lactobacillus strains inhibited $S$. mutans growth and biofilm formation

\footnotetext{
* Corresponding author.

E-mail addresses: dennisrossoni@hotmail.com (R.D. Rossoni), marisol_velloso2@hotmail.com (M.d.S. Velloso), barrosdnapp@yahoo.com.br (P.P.de Barros), janaina_2406@hotmail.com (J.A.de Alvarenga), jessicadiane.santos@yahoo.com.br (J.D.d. Santos), anacarolinachipoletti@hotmail.com (A.C.C.d. Santos Prado), felipe_c_ribeiro@hotmail.com (F.d.C. Ribeiro), analia@ict.unesp.br (A.L. Anbinder), juliana@ict.unesp.br (J.C. Junqueira).

1 These authors contributed equally to this work.
} 
on glass surfaces, but the effects depended on the Lactobacillus strains used.

These studies suggested that several strains used in dietary products can inhibit $S$. mutans, showing potential for their use as probiotics to prevent dental caries $[10,13,14]$. However, most commercially available probiotic strains were isolated from human or animal gastrointestinal tracts $[5,11]$. Thus, their beneficial effects for preventing dental caries and other oral diseases may be questionable $[8,15]$. The identification of new Lactobacillus strains from the oral cavity that can inhibit $S$. mutans is essential for the development of the use of probiotics for the prevention of dental caries. Our study was based on the hypothesis that the oral cavities of healthy individuals may harbor some beneficial Lactobacillus strains that can release bioactive substances with inhibitory activities against oral pathogens.

Most of the studies cited above focused on the use of living cells of probiotics; however, the effects of Lactobacillus supernatants from the oral cavity on $S$. mutans biofilms have been studied very little. Thus, in order to understand the effects of exometabolites produced by Lactobacillus and to explore the possible application of the secondary metabolites on oral biofilms in the future, in this study, we evaluated the antibiofilm effects of cell-free supernatants of Lactobacillus strains from the oral cavity on $S$. mutans.

\section{Materials and methods}

\subsection{Microorganisms and growth conditions}

In this study, we used Streptococcus mutans UA159 and 22 clinical strains of Lactobacillus that were previously isolated from the oral cavities of caries-free subjects, which were identified by an API $50 \mathrm{CHL}$ system (BioMérieux, France) and confirmed by PCR. These Lactobacillus strains showed great potential to inhibit $C$. albicans strains from the oral cavity in vitro and to negatively modulate virulence gene expression [16]. This study was approved by the Human Research Ethics Committee of the Institute of Science and Technology, Univ Estadual Paulista/UNESP (protocol number 754.634).

\subsection{Preparation of microbial inocula and culture filtrates}

S. mutans was grown in Brain Heart Infusion broth (BHI broth, Himedia, Mumbai, India), and the Lactobacillus strains were grown in DeMan-Rogosa-Sharpe broth (MRS broth, Difco, Detroit, USA) at $37^{\circ} \mathrm{C}$ for $48 \mathrm{~h}\left(5 \% \mathrm{CO}_{2}\right)$. The microbial cells in culture were centrifuged, and the pellets were washed twice with $0.85 \% \mathrm{NaCl}$ (Labimpex, São Paulo, Brazil). The cell suspensions were adjusted to $10^{8}$ cells $/ \mathrm{mL}$ using a spectrophotometer (B582, Micronal, Brazil).

To prepare culture filtrates of the Lactobacillus strains, $1 \mathrm{~mL}$ of a $10^{8}$ cells/mL Lactobacillus culture (previously prepared) was added to MRS broth and was incubated at $37^{\circ} \mathrm{C}$ for $24 \mathrm{~h}\left(5 \% \mathrm{CO}_{2}\right)$. Next, the culture was centrifuged and the supernatant was filtered through a $0.22-\mu \mathrm{m}$ filter (MFS, Dublin, USA).

\subsection{Antibacterial activity of Lactobacillus supernatants against $S$. mutans in planktonic cultures}

The antibacterial activity of the Lactobacillus strains against $S$. mutans in planktonic cultures was assessed according to the methodology described by Lin et al. [10] with some modifications. Standardized $S$. mutans and Lactobacillus cell suspensions were prepared as described above. Next, $250 \mu \mathrm{L}$ of a $S$. mutans suspension and $250 \mu \mathrm{L}$ of the Lactobacillus supernatant were mixed with $1.5 \mathrm{~mL}$ of BHI broth. In the control group, the microbial suspension of $S$. mutans was cultured with a physiological solution. All of the cultures were incubated at $37^{\circ} \mathrm{C}$ for 12 and $24 \mathrm{~h}\left(5 \% \mathrm{CO}_{2}\right)$. After incubation, the cultures were diluted and plated on Mitis Salivarius Agar (Difco, Detroit, USA) supplemented with bacitracin (Sigma-Aldrich, St. Paul, Brazil, $0.2 \mathrm{IU} / \mathrm{mL}$ ) and 15\% sucrose
(MSBS) for $S$. mutans growth. The plates were incubated at $37{ }^{\circ} \mathrm{C}$ for $48 \mathrm{~h}\left(5 \% \mathrm{CO}_{2}\right)$, and the number of colony-forming units ( $\left.\mathrm{CFU} / \mathrm{mL}\right)$ was determined. This assay was performed as three independent experiments with four replicates per group.

\subsection{Antibacterial activity of Lactobacillus supernatants on S. mutans counts in biofilms}

To form biofilms, we used hydroxyapatite discs $(5 \mathrm{~mm}$ diameter $\mathrm{x}$ $2 \mathrm{~mm}$ thick) purchased from Clarkson Chromatography Products, Inc. (South Williamsport, USA) following the methodology described by Marttinen et al. [14] with modifications. The sterilized discs were placed in 24-well culture plates (Kasvi, Curitiba, Brazil) containing $1.8 \mathrm{~mL}$ of a mixture composed of $70 \%$ saliva and $30 \%$ BHI broth supplemented with $0.3 \%$ glucose and $67 \mathrm{mmol} / \mathrm{L}$ Sörensen's buffer $(\mathrm{pH}$ 7.2). A $225-\mu \mathrm{L}$ aliquot of a standardized suspension of $S$. mutans was added, and the plates were incubated at $37^{\circ} \mathrm{C}$ for $1 \mathrm{~h}\left(5 \% \mathrm{CO}_{2}\right)$ to promote the initial adhesion of $S$. mutans onto the discs. Next, $225 \mu \mathrm{L}$ of Lactobacillus supernatant was added. In the control group, the microbial suspensions of $S$. mutans were cultivated with physiological solution. For this experiment, $S$. mutans cells were counted in biofilms formed in $24 \mathrm{~h}$ and in $48 \mathrm{~h}$.

After incubation ( 24 or $48 \mathrm{~h}$ ), the discs were washed 3 times and transferred to a tube containing $1 \mathrm{~mL}$ of a $\mathrm{NaCl}$ solution. The biofilms formed were detached using an ultrasonic homogenizer (Sonopuls HD 2200, Bandelin Electronic) at $7 \mathrm{~W}$ for $30 \mathrm{~s}$. The suspensions were serially diluted and plated on MSBS agar to determine the number of CFU/ $\mathrm{mL}$. The biofilm experiments were performed in three independent experiments on different days with four biofilms per group. For the biofilm group formed in $48 \mathrm{~h}$, after $24 \mathrm{~h}$ of incubation, the discs were washed 3 times with a $\mathrm{NaCl}$ solution and transferred into a fresh medium mixture composed of $1.8 \mathrm{~mL}$ of $70 \%$ saliva and $30 \%$ BHI broth supplemented with $0.15 \%$ glucose and $0.15 \%$ sucrose.

\subsection{Measurement of $\mathrm{pH}$ values}

The $\mathrm{pH}$ values of the media were tested during the biofilm formation under the same conditions as the biofilm assay described above. After $48 \mathrm{~h}$ of incubation in the 24-well culture plates, the supernatants from each well were collected, and the $\mathrm{pH}$ values were measured using a pH meter (Mettler, Toledo, Ohio, USA). Four wells were measured per group, and the experiment was done at three different times.

\subsection{Analysis of biofilms by scanning electron microscopy (SEM)}

In this experiment, we used hydroxyapatite discs ( $5 \mathrm{~mm}$ diameter $\mathrm{x}$ $2 \mathrm{~mm}$ thick), and the biofilms were formed as mentioned above. After biofilm formation, the specimens were fixed in $1 \mathrm{~mL}$ of $2.5 \%$ glutaraldehyde for $1 \mathrm{~h}$. The specimens were then dehydrated in an increasing ethanol concentration series (10, 25, 50, 75 and 90\%) for $20 \mathrm{~min}$ each, followed by immersion in $100 \%$ alcohol for $1 \mathrm{~h}$. The plates were kept in an oven at $37^{\circ} \mathrm{C}$ for $24 \mathrm{~h}$ to permit total drying of the specimens.

After drying, the specimens were transferred to aluminum stubs and sputter coated with gold for $160 \mathrm{~s}$ at $40 \mathrm{~mA}$ (Denton Vacuum Desk II, Denton Vacuum LLC, Moorestown, NJ, USA). The specimens were examined and imaged using a JEOL JSM-5600 scanning electron microscope (JEOL USA, Inc., Peabody, MA, USA) at the Institute of Science and Technology, UNESP - Univ Estadual Paulista. These experiments were performed at two different times with $n=3$ biofilms per group.

\subsection{Antibacterial activity of Lactobacillus supernatants on S. mutans biofilm biomass}

After biofilm formation, the biofilm biomass was quantified utilizing an assay previously described by Rossoni et al. [16] and Peeters et al. [17], with modifications. For fixation of the biofilms, $100 \mu l$ of 
99\% methanol was added to the wells (Sigma-Aldrich, São Paulo, Brazil). After $15 \mathrm{~min}$, the supernatants were removed and the plates were air-dried.

Then, $100 \mu$ of a $1 \%$ crystal violet (CV) solution was added to all wells. After $20 \mathrm{~min}$, the residual CV solution was removed by washing with PBS. Finally, bound CV was released by adding $150 \mu \mathrm{l}$ of $33 \%$ acetic acid (Sigma-Aldrich). The absorbance was measured at $540 \mathrm{~nm}$. All steps were carried out at room temperature. The CV assay was performed as two independent experiments with $n=6$ biofilms per group.

\subsection{Statistical analysis}

The results were compared by ANOVA and Tukey's test. Student's $t$ test was used to compare the $\mathrm{CFU} / \mathrm{mL}$ results from the in vitro biofilm formation assay and the CV assay. All analyses were performed using the GraphPad Prism 6 Program (GraphPad Software, Inc., La Jolla, CA, USA), and a level of significance of $5 \%$ was adopted.

\section{Results}

We selected 22 clinical strains of Lactobacillus that were previously isolated from the oral cavities of caries-free subjects, including 22 strains of $L$. paracasei $(1.1,3.1,6.2,7.5,8.4,11.6,15.8,16.4,17.1$, 20.3, 21.4, 23.4, 24.1, 25.4, 26.1, 27.1, 28.4 and 30.1), 3 strains of $L$. fermentum (14.5, 20.4 and 31.4) and 1 strain of L. rhamnosus (19.3). All of the strains were screened for antibacterial activity against $S$. mutans using planktonic cultures. For this purpose, we analyzed the indirect effects of Lactobacillus using only the Lactobacillus culture filtrate that was obtained after its growth in MRS broth (S. mutans + Lactobacillus supernatant interaction group) for 12 and $24 \mathrm{~h}$. As a control, we also tested monocultures of $S$. mutans ( $S$. mutans + physiological solution control group). To determine whether the MRS broth of the Lactobacillus culture could exert an effect on $S$. mutans and interfere with the results, we included a control group consisting only of $S$. mutans and MRS broth (S. mutans + MRS broth control group) (data not shown). After 12 or $24 \mathrm{~h}$ in culture, the $S$. mutans growth was evaluated by determining the colony-forming unit numbers $(\mathrm{CFU} / \mathrm{mL})$.

All 22 Lactobacillus strains (100\%) analyzed showed antibacterial activity against $S$. mutans. Only the $L$. paracasei $8.4,23.4$ and 24.1 strains had no inhibitory effects on $S$. mutans after $24 \mathrm{~h}$ in culture. We observed a reduction in the number of $S$. mutans cells in the $S$. mutans + Lactobacillus interaction group compared to the $S$. mutans + physiological solution control group $(100 \%$ of $S$. mutans growth). The reductions in $S$. mutans growth ranged from 12 to $91.4 \%$ depending on the strain analyzed (Fig. 1).

The results showed that the MRS broth used to prepare the Lactobacillus supernatant did not interfere with the growth of $S$. mutans.
The CFU/mL count of $S$. mutans was $10.02 \pm 0.06$ for the $S$. mutans + physiological solution control group and $10.04 \pm 0.03$ for the $S$. mutans + MRS broth control group (Student's $t$-test, $p=0.44$ ). These data indicated that the anti-S. mutans activity of the supernatants could be attributed to metabolites produced by the Lactobacillus strains.

Based on the results presented in Fig. 1, we can observe that the four strains with the highest antibacterial activity against $S$. mutans were $L$. paracasei 25.4, L. fermentum 20.4 , L. paracasei 20.3 and L. paracasei 11.6. These strains reduced $S$. mutans growth by more than $86 \%$ after $24 \mathrm{~h}$ in culture. Therefore, these strains were selected for the in vitro $S$. mutans biofilm studies.

The $S$. mutans biofilms were formed on hydroxyapatite discs, and after incubation times of 24 and $48 \mathrm{~h}$, the biofilms were analyzed by counting the $\mathrm{CFU} / \mathrm{mL}$. In these experiments, we found a strong inhibitory activity of Lactobacillus supernatant on $S$. mutans cells for all time points tested. For all of the Lactobacillus supernatants tested (11.6, 20.4, 20.3 and 25.4), there were statistically significant differences between the $S$. mutans + physiological solution control group and the S. mutans + Lactobacillus supernatant interaction group (Fig. 2). $L$. paracasei strain 25.4 presented the largest reduction (time points: $24 \mathrm{~h}$ $3.73 \mathrm{log}$ and $48 \mathrm{~h} 3.49 \mathrm{log}$ ) in the number of viable $S$. mutans cells determined by the CFU count. These findings indicated that these Lactobacillus strains release bioactive substances that can inhibit $S$. mutans growth and biofilm formation. Due to the great clinical importance of mature $S$. mutans biofilms in caries formation, and as we demonstrated the efficiency of the Lactobacillus supernatants in the early and late stages of $S$. mutans biofilm formation, we decided to evaluate the interference of the $\mathrm{pH}$ levels of these supernatants on the biofilms, and the total biomass and SEM analysis were performed only on biofilms formed after $48 \mathrm{~h}$ of incubation.

To investigate whether the effects of Lactobacillus supernatants on $S$. mutans were associated with $\mathrm{pH}$ variations, we monitored the $\mathrm{pH}$ values of the culture media at the same time point as for the biofilm growth $(48 \mathrm{~h})$. For all of the experimental groups, the $\mathrm{pH}$ values decreased from 7.2 (initial biofilm $\mathrm{pH}$ ) to between 4 and 5 , and no major $\mathrm{pH}$ variations were observed at the different times of biofilm growth (Fig. 3). Since the $\mathrm{pH}$ values in the $S$. mutans + PBS control group were similar to the $S$. mutans + Lactobacillus supernatant interaction groups, we concluded that $\mathrm{pH}$ variation is not a crucial factor in the inhibitory activity of Lactobacillus supernatants on $S$. mutans biofilms.

The biofilms formed were also evaluated by SEM, and with this approach, we can observe mature biofilm formation on hydroxyapatite discs. The $S$. mutans cells observed in the biofilms had different growth features depending on the experimental group. The biofilms formed by $S$. mutans in the absence of Lactobacillus supernatant were characterized by the presence of numerous bacterial cells and formation of an extracellular matrix (Fig. 4A and B). In the "S. mutans + Lactobacillus supernatant interaction group", we verified a reduction in the number

\section{Antibacterial Activity}

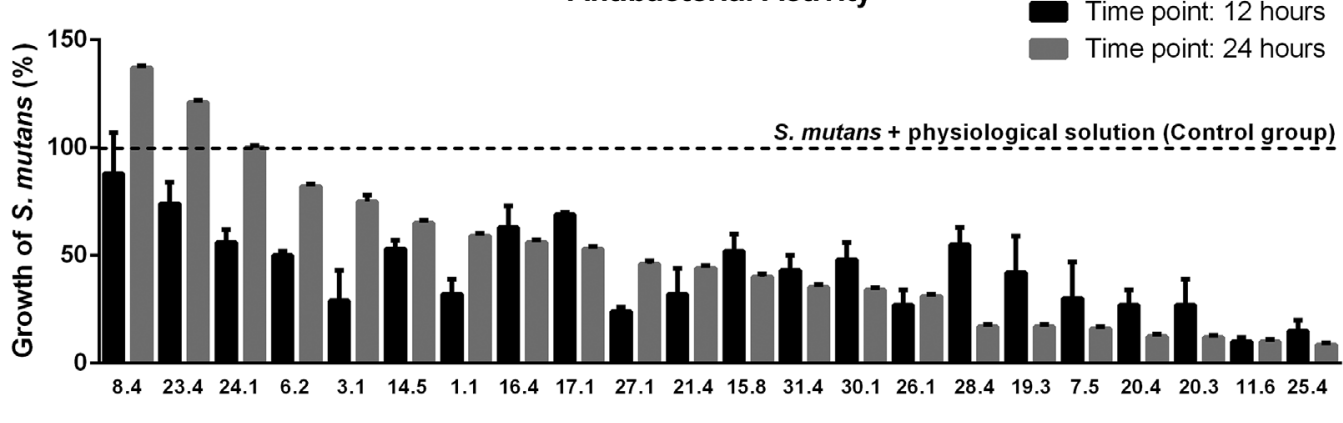

Lactobacillus strains
Fig. 1. Growth percentages of $S$. mutans obtained by counting the $\mathrm{CFU} / \mathrm{mL}$ in the antibacterial activity in vitro test for 12 and $24 \mathrm{~h}$ in the presence of Lactobacillus supernatants. The supernatants of 22 Lactobacillus strains were tested: 22 strains of $L$. paracasei $(1.1,3.1,6.2,7.5,8.4,11.6$, $15.8,16.4,17.1,20.3,21.4,23.4,24.1$, 25.4, 26.1, 27.1, 28.4 and 30.1), 3 strains of $L$. fermentum (14.5, 20.4 and 31.4) and 1 strain of L. rhamnosus (19.3). The graph shows the percentage of $S$. mutans growth in the "S. mutans + Lactobacillus supernatant interaction group (gray and black bars)" in relation to the "S. mutans + phy siological solution control group (-)" (100\% of S. mutans growth). 
A

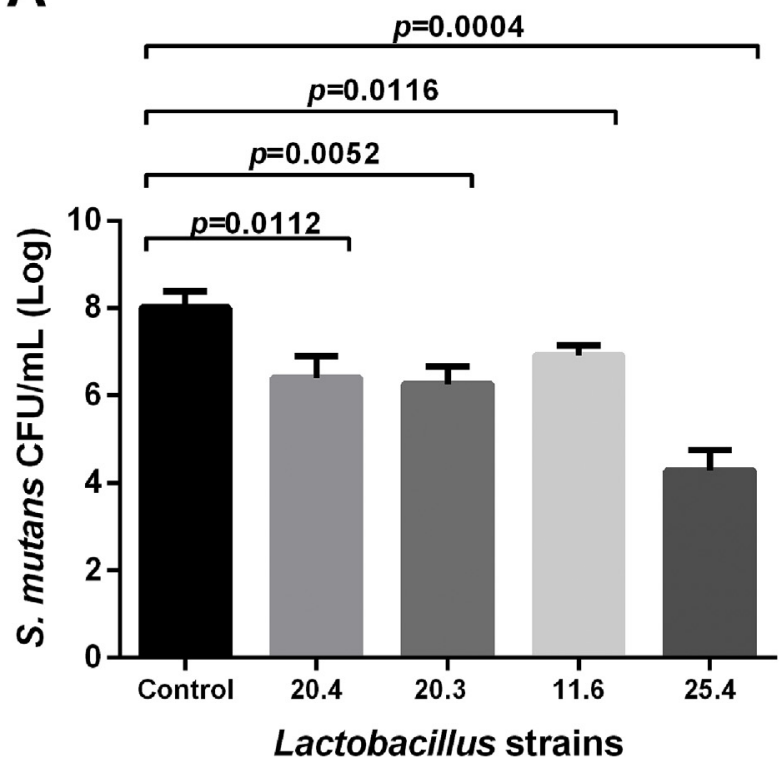

Time point: 24 hours

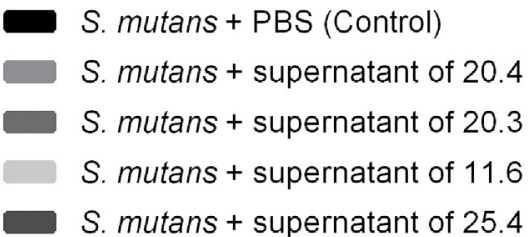

S. mutans + PBS (Control)

S. mutans + supernatant of 20.4

20.3

S. mutans + supernatant of 25.4
B

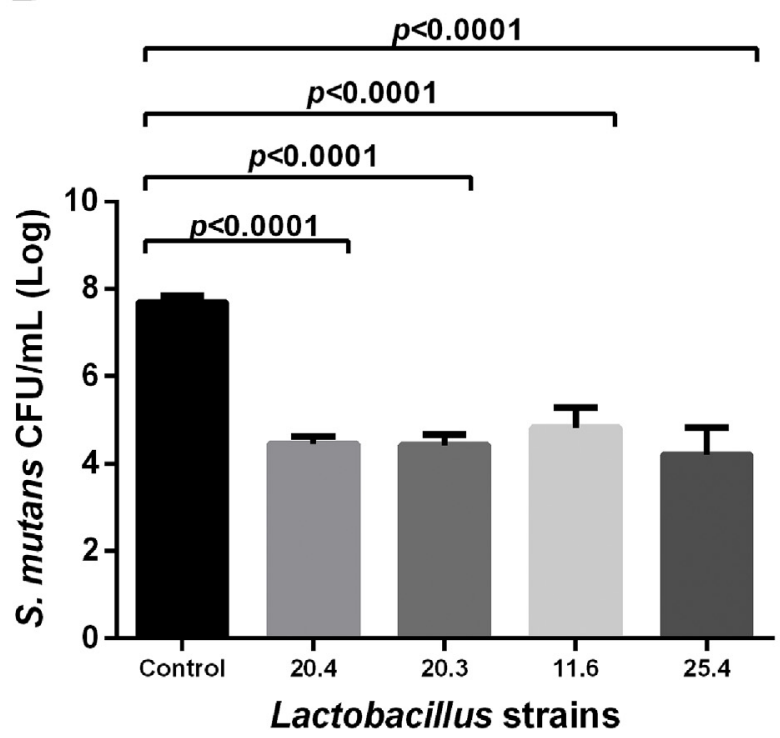

Time point: 48 hours

S. mutans + PBS (Control)

S. mutans + supernatant of 20.4

S. mutans + supernatant of 20.3

S. mutans + supernatant of 11.6

S. mutans + supernatant of 25.4

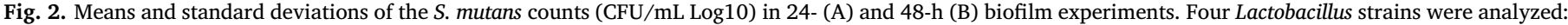

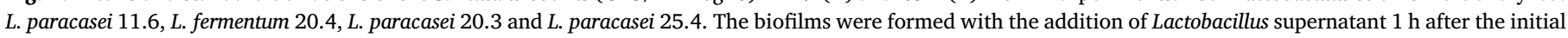

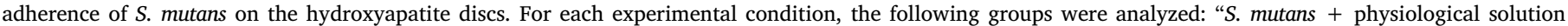
control group" and "S. mutans + Lactobacillus supernatant interaction group". For Student's $t$-test, the differences were considered significant at $p \leq 0.05$.

\section{Time of biofilm formation ( $48 \mathrm{~h}$ )}

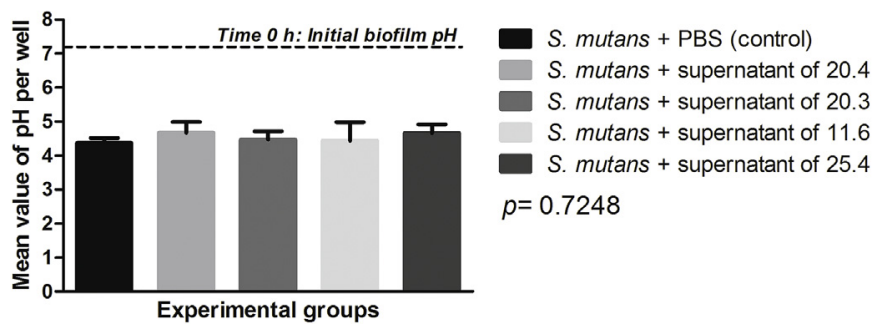

Fig. 3. Means and standard deviations of $\mathrm{pH}$ values obtained in the biofilm formation assay. For each Lactobacillus supernatant studied, the following groups were analyzed: "S. mutans + physiological solution control group" and "S. mutans + Lactobacillus supernatant interaction group". of $S$. mutans cells and less extracellular matrix formation compared to the control group (Fig. 4C-F). Therefore, SEM images confirmed the results obtained from the $\mathrm{CFU}$ count, showing that the supernatants of Lactobacillus isolates influenced $S$. mutans viability and reduced the total biofilm biomass.

In order to confirm that the Lactobacillus supernatants also influence the extracellular matrix formation of $S$. mutans biofilms, the total biomass was quantified by the colorimetric assay using CV. Prioritizing the Lactobacillus supernatants that reduced $S$. mutans growth most significantly in the biofilm, we also used the following four strains: $L$. paracasei 11.6, L. paracasei 20.3 , L. fermentum 20.4 and L. paracasei 25.4. The biofilms formed by $S$. mutans in the presence of Lactobacillus supernatants exhibited a significant reduction compared to the $S . \mathrm{mu}$ tans control group (Fig. 5). 

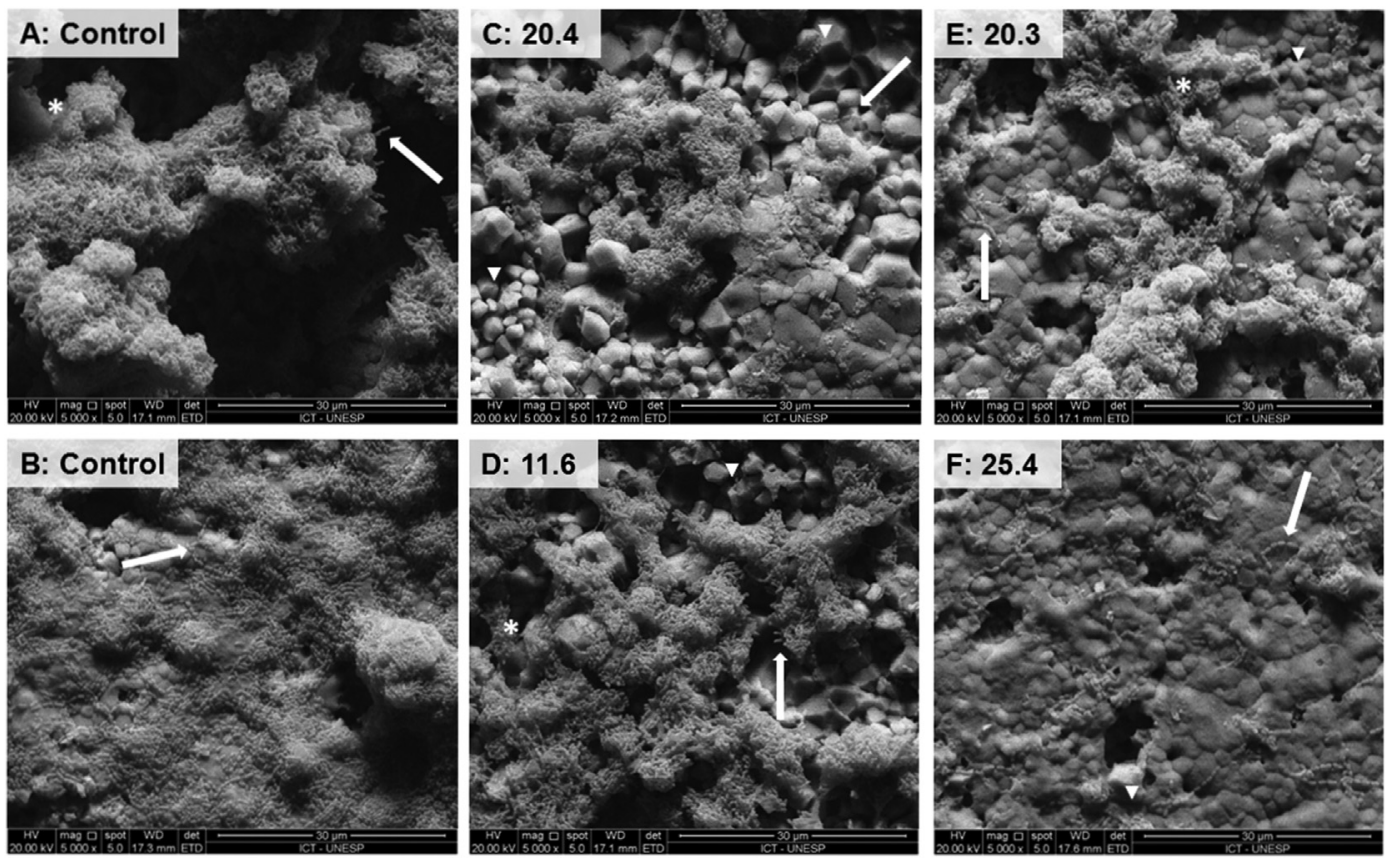

Fig. 4. SEM of biofilms formed in vitro. A. and B. Control group of $S$. mutans + physiological solution; the presence of numerous bacterial cells and formation of extracellular matrix is verified; C. Group interaction of $S$. mutans + supernatant of L. fermentum 20.4; D. Group interaction of $S$. mutans + supernatant of $L$. paracasei 11.6; E. Group interaction of $S$. mutans + supernatant of $L$. paracasei 20.3; F. Group interaction of $S$. mutans + supernatant of $L$. paracasei 25.4. In all of the interaction biofilms, it was possible to observe a reduction in the number of $S$. mutans cells compared to the control group. $S$. mutans cells (arrow), extracellular matrix (asterisk) and hydroxyapatite crystals (filled inverted triangle) are highlighted in the SEM. Magnification: 5000X.

Considering the results observed from the CV assay, we confirm the inhibitory effect of probiotic Lactobacillus supernatants from the oral cavity on $S$. mutans biofilm. In addition, this antibiofilm effect can be attributed to the reduction in the number of $S$. mutans cells and the lower extracellular matrix formation. $L$. paracasei strain 25.4 is a potential probiotic candidate for clinical studies focused on the prevention of dental caries.

\section{Discussion}

In recent decades, the interest in using probiotics to prevent oral infectious diseases has grown significantly [18-20]. Certain Lactobacillus strains have shown a potential ability to specifically interfere with oral ecology by inhibiting pathogenic microorganisms, such as $S$. $m u$ tans $[10,11,21-23]$. Therefore, the use of probiotic lactobacilli seems to be a promising method for controlling dental caries [24]. Based on these observations, the aim of our study was to identify new Lactobacillus strains and to test whether only its supernatant, with their associated active metabolites, can be safely used as prophylactic agents in the oral cavity to inhibit $S$. mutans growth and biofilm formation. The use of the supernatant alone is safer than using live Lactobacillus cells in relation to caries formation because some strains of this bacterial genus can metabolize sucrose, co-aggregate with $S$. mutans and are often tolerant toward the use of fluoride $[25,26]$.

It has been suggested that individuals who have never had dental caries may harbor Lactobacillus strains in their oral cavities that contribute to a healthy microbiota and to the control of dental caries $[11,27]$. Thus, we screened 22 supernatants from different Lactobacillus strains isolated from the oral cavities of caries-free subjects, including several strains of $L$. paracasei, L. rhamnosus and L. fermentum. Using in

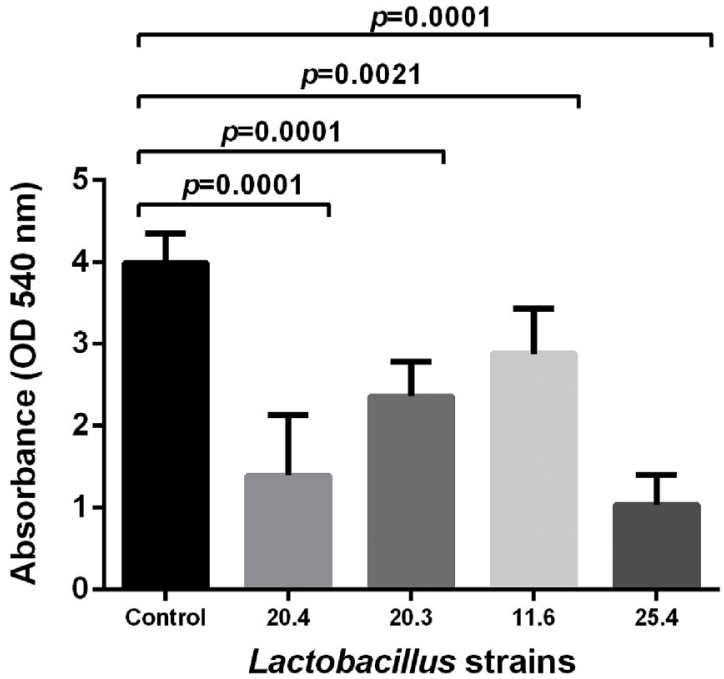

Fig. 5. Evaluation of the biomass of $S$. mutans biofilms. Means and standard deviations of the absorbance values of the control group biofilms ("S. mutans + physiological solution control group") and in the groups with Lactobacillus supernatants "S. mutans + Lactobacillus supernatant interaction group". Four Lactobacillus strains were analyzed: L. paracasei $11.6, L$. fermentum 20.4, L. paracasei 20.3 and L. paracasei 25.4. For Student's $t$-test, differences were considered significant at $p \leq 0.05$. 
vitro planktonic cultures, we identified supernatants of four strains ( 3 strains of $L$. paracasei and 1 of $L$. fermentum) capable of limiting $S$. mutans growth by more than $86 \%$.

Our findings agree with the study of Simark-Mattsson et al. [11] in which they isolated Lactobacillus strains from caries-free subjects and from individuals with active caries. The antimicrobial activities of these strains against $S$. mutans were evaluated using agar overlay interference tests. The authors observed that the Lactobacillus strains isolated from caries-free subjects had higher inhibitory activities against $S$. mutans than did the Lactobacillus strains isolated from individuals with active caries. Among the Lactobacillus strains isolated from caries-free subjects, 23 could completely inhibit the growth of $S$. mutans, and the species with the highest antibacterial activities included $L$. paracasei, $L$. plantarum and L. rhamnosus.

Next, we used in vitro biofilm models to determine whether the antibacterial activities of the assayed Lactobacillus supernatants act upon $S$. mutans cells organized in biofilms. The use of biofilm models to study the effects of antibacterial agents against $S$. mutans is very important, since the cariogenic activity of $S$. mutans is largely attributed to its ability to adhere to teeth and to create a molecular scaffold of glucan polysaccharides on the tooth surfaces [28]. Several laboratories have developed in vitro biofilm models that can mimic the oral cavity environment [29]. In the present study, we evaluated two biofilm methods: the first was to form biofilms on hydroxyapatite discs to simulate the tooth surface that were placed in contact with artificial saliva to induce a salivary pellicle formation [30], and the second approach was to form biofilms at the bottom of wells to evaluate the total biomass. Using the first biofilm method, we verified that the Lactobacillus supernatants could interfere with $S$. mutans biofilms, leading to a reduction in their numbers of $\mathrm{CFU} / \mathrm{mL}$. These results validated the hypothesis that the antimicrobial activity of Lactobacillus supernatants against oral pathogens in biofilms can be attributed to the production of bioactive substances [31,32].

Recently, Krzyściak et al. [33] evaluated the anti-cariogenic effects of Lactobacillus salivarius CECT5713 by limiting S. mutans and C. albicans growth and biofilm mass in a double-species biofilm model. The authors found that $L$. salivarius inhibited the cariogenic biofilm formation of $C$. albicans and $S$. mutans. Under the influence of the probiotic, the biofilm mass and the number of $S$. mutans colonies in the biofilm were decreased (about $0.15 \log$ reduction). These results corroborate the reduction of $S$. mutans described in the present study (3.73 $\log$ for $L$. paracasei 25.4).

To assess the influence of the acids produced by Lactobacillus strains on $S$. mutans, which consequently lower the $\mathrm{pH}$ of the supernatant, we measured the $\mathrm{pH}$ values of biofilm formation of each Lactobacillus supernatant. All of the Lactobacillus strains had similar acidogenic activities; therefore, no major $\mathrm{pH}$ variations were observed at the different times of biofilm growth compared to the control group. However, some previous studies demonstrated that the acidic environment provided by Lactobacillus strains can directly affect their antibacterial activities against $S$. mutans [10,22]. Keller et al. [22] evaluated the ability of commercial probiotic lactobacilli to co-aggregate and to inhibit the growth of $S$. mutans. The selected lactobacilli showed co-aggregation activity and inhibited the growth of $S$. mutans according to variations in $\mathrm{pH}$. Therefore, the role of the acids produced by probiotic strains on $S$. mutans growth is still unclear. In addition to acids, the production of antimicrobial compounds by probiotic bacteria has also been widely discussed. Previous studies showed that Lactobacillus strains produce bacteriolytic enzymes, bacteriocins and biosurfactants that can inhibit pathogenic microorganisms [34-36]. Some bacteriocins produced by lactobacilli have been purified and extensively studied, such as gasserin produced by L. gasseri EV1461 [34], fermecin produced by L. fermentum SD11 [35], and paracin 1.7 produced by L paracasei HD1-7 [36].

The biofilms were also evaluated by SEM analysis in which $S$. mutans is strongly adherent on hydroxyapatite discs. In addition, it was possible to verify a reduction in the number of $S$. mutans cells when the control biofilm was compared to the biofilms exposed to the Lactobacillus supernatants. According to the SEM analysis, L. paracasei 25.4 (the strain with the greatest reduction in $\mathrm{CFU} / \mathrm{mL}$ count) reduced the adhesion of $S$. mutans cells to the discs, and this effect probably caused the reduction in the CFU count. The images obtained in this study are in agreement with Wasfi et al. [37], who investigated whether L. casei (ATCC 393), L. reuteri (ATCC 23272), L. plantarum (ATCC 14917) or L. salivarius (ATCC 11741) inhibit $S$. mutans biofilms. In their SEM images, the authors observed changes in the extracellular matrix, fewer bacteria and smaller microcolonies in biofilms formed during coculture of $S$. mutans and Lactobacillus.

Using the second biofilm evaluation method, we quantified the differences in the total biomass of the biofilms among the groups, and in this approach, the biofilms were stained with CV. The biofilms formed by $S$. mutans in the presence of Lactobacillus supernatants exhibited a significant reduction in biomass compared to the control group that lacked Lactobacillus supernatant. All of the supernatants tested significantly reduced the biomass, and $L$. paracasei 25.4 was the strain with the highest capacity to affect $S$. mutans biofilms. Ahn et al. [38] also demonstrated that $L$. plantarum lipoteichoic acid could inhibit the biofilm formation of $S$. mutans on polystyrene plates stained with $\mathrm{CV}$ and on hydroxyapatite discs.

\section{Conclusion}

In conclusion, this study showed that most Lactobacillus strains isolated from the oral cavities of caries-free subjects could release bioactive substances that inhibit the growth of $S$. mutans in planktonic cultures. The strains $L$. paracasei 11.6, L paracasei 25.4, L. fermentum 20.4 and $L$. paracasei 20.3 presented antibiofilm activity against $S$. mutans in different methods of biofilm quantification. The identification of these Lactobacillus strains, which naturally inhabit the oral cavity and show antimicrobial activity against $S$. mutans, contributes to the development of new probiotic agents to prevent dental caries.

\section{Conflicts of interest}

The authors declare that they have no conflicts of interest.

\section{Acknowledgements}

This study was supported by the São Paulo Council of Research FAPESP, Brazil (Grants 2013/25181-8, 2014/11841-9, 2015/09770-9 and 2017/19219-3) and by "National Council for Scientific and Technological Development - CNPq"

\section{References}

[1] X. He, R. Lux, H.K. Kuramitsu, M.H. Anderson, W. Shi, Achieving probiotic effects via modulating oral microbial ecology, Adv. Dent. Res. 21 (2009) 53-56.

[2] Y. Su, B. Zhang, L. Su, CD4 detected from Lactobacillus helps understand the interaction between Lactobacillus and HIV, Microbiol. Res. 168 (2013) 273-277.

[3] L.A. Gonzalez-Hernandez, L.F. Jave-Suarez, M. Fafutis-Morris, K.E. Montes-Salcedo, L.G. Valle-Gutierrez, A.E. Campos-Loza, et al., Synbiotic therapy decreases microbial translocation and inflammation and improves immunological status in HIVinfected patients: a double-blind randomized controlled pilot trial, Nutr. J. 11 (2012) 90 .

[4] S.F. Vilela, J.O. Barbosa, R.D. Rossoni, J.D. Santos, M.C. Prata, A.L. Anbinder, et al., Lactobacillus acidophilus ATCC 4356 inhibits biofilm formation by C. albicans and attenuates the experimental candidiasis in Galleria mellonella, Virulence 6 (2015) 29-39.

[5] Y. Rivera-Espinoza, Y. Gallardo-Navarro, Non-dairy probiotic products, Food Microbiol. 27 (2010) 1-11.

[6] F. Shanahan, T.G. Dinan, P. Ross, C. Hill, Probiotics in transition, Clin. Gastroenterol. Hepatol. 10 (2012) 1220-1224.

[7] B. Olle, Medicines from microbiota, Nat. Biotechnol. 31 (2013) 309-315.

[8] K. Pradeep, M.A. Kuttappa, K.R. Prasana, Probiotics and oral health: an update, SADJ 69 (2014) 20-24.

[9] A. Haukioja, Probiotics and oral health, Eur. J. Dermatol. 4 (2010) 348-355.

[10] X. Lin, X. Chen, Y. Chen, W. Jiang, H. Chen, The effect of five probiotic lactobacilli strains on the growth and biofilm formation of Streptococcus mutans, Oral Dis. 21 
(2015) e128-e134.

[11] C. Simark-Mattsson, C. G. Emilson, E. G. Hakansson, C. Jacobsson, K. Roos, S. Holm, Lactobacillus-mediated interference of mutans streptococci in caries-free vs. cariesactive subjects, Eur. J. Oral Sci. 115 (2007) 308-314.

[12] S. Piwat, B. Sophatha, R. Teanpaisan, An assessment of adhesion, aggregation and surface charges of Lactobacillus strains derived from the human oral cavity, Lett. Appl. Microbiol. 61 (2015) 98-105.

[13] E.M. Soderling, A.M. Marttinen, A.L. Haukioja, Probiotic lactobacilli interfere with Streptococcus mutans biofilm formation in vitro, Curr. Microbiol. 62 (2011) 618-622.

[14] A.M. Marttinen, A.L. Haukioja, M. Keskin, E.M. Soderling, Effects of Lactobacillus reuteri PTA 5289 and L. paracasei DSMZ16671 on the adhesion and biofilm formation of Streptococcus mutans, Curr. Microbiol. 67 (2013) 193-199.

[15] P. Sornplang, S. Piyadeatsoontorn, Probiotic isolates from unconventional sources: a review, J. Anim. Sci. Technol. 58 (2016) 26.

[16] R.D. Rossoni, P.P. de Barros, J.A. de Alvarenga, F.C. Ribeiro, M.D.S. Velloso, B.B. Fuchs, et al., Antifungal activity of clinical Lactobacillus strains against Candida albicans biofilms: identification of potential probiotic candidates to prevent oral candidiasis, Biofouling 34 (2018) 212-225.

[17] E. Peeters, H.J. Nelis, T. Coenye, Comparison of multiple methods for quantification of microbial biofilms grown in microtiter plates, J. Microbiol. Meth. 72 (2008) $157-165$.

[18] J.M. Tanzer, A. Thompson, C. Lang, B. Cooper, L. Hareng, A. Gamer, et al., Caries inhibition by and safety of Lactobacillus paracasei DSMZ16671, J. Dent. Res. 89 (2010) 921-926.

[19] S. Twetman, M.K. Keller, Probiotics for caries prevention and control, Adv. Dent Res. 24 (2012) 98-102.

[20] N. Yanine, I. Araya, R. Brignardello-Petersen, A. Carrasco-Labra, A. Gonzalez, A. Preciado, et al., Effects of probiotics in periodontal diseases: a systematic review, Clin. Oral Invest. 17 (2013) 1627-1634.

[21] P. Hasslof, M. Hedberg, S. Twetman, C. Stecksen-Blicks, Growth inhibition of ora mutans streptococci and candida by commercial probiotic lactobacilli-an in vitro study, BMC Oral Health 10 (2010) 18.

[22] M.K. Keller, P. Hasslof, C. Stecksen-Blicks, S. Twetman, Co-aggregation and growth inhibition of probiotic lactobacilli and clinical isolates of mutans streptococci: an in vitro study, Acta Odontol. Scand. 69 (2011) 263-268.

[23] P.L. Holgerson, N.R. Vestman, R. Claesson, C. Ohman, M. Domellof, A.C. Tanner, et al., Oral microbial profile discriminates breast-fed from formula-fed infants, J. Pediatr. Gastroenterol. Nutr. 56 (2013) 127-136.

[24] M.G. Cagetti, S. Mastroberardino, E. Milia, F. Cocco, P. Lingstrom, G. Campus, The use of probiotic strains in caries prevention: a systematic review, Nutrients 5 (2013) $2530-2550$.

[25] S. Saha, C. Tomaro-Duchesneau, L. Rodes, M. Malhotra, M. Tabrizian, S. Prakash,
Investigation of probiotic bacteria as dental caries and periodontal disease biotherapeutics, Benef. Microbes 5 (2014) 447-460.

[26] P.W. Caufield, C.N. Schon, P. Saraithong, Y. Li, S. Argimon, Oral Lactobacilli and dental caries: a model for niche adaptation in humans, J. Dent. Res. 94 (2015) 110S-8S.

[27] P. Belda-Ferre, L.D. Alcaraz, R. Cabrera-Rubio, H. Romero, A. Simon-Soro, M. Pignatelli, et al., The oral metagenome in health and disease, ISME J. 6 (2012) $46-56$.

[28] J.M. Ansari, N.M. Abraham, J. Massaro, K. Murphy, J. Smith-Carpenter, E. Fikrig, Anti-biofilm activity of a self-aggregating peptide against Streptococcus mutans, Front. Microbiol. 8 (2017) 488.

[29] K.M. Salli, U.K. Gursoy, E.M. Soderling, A.C. Ouwehand, Effects of xylitol and sucrose mint products on Streptococcus mutans colonization in a dental simulator model, Curr. Microbiol. 74 (10) (2017) 1153-1159.

[30] J. Samot, J. Lebreton, C. Badet, Adherence capacities of oral lactobacilli for potential probiotic purposes, Anaerobe 17 (2011) 69-72.

[31] V.H. Matsubara, Y. Wang, H.M. Bandara, M.P. Mayer, L.P. Samaranayake, Probiotic lactobacilli inhibit early stages of Candida albicans biofilm development by reducing their growth, cell adhesion, and filamentation, Appl. Microbiol. Biotechnol. 100 (2016) 6415-6426.

[32] F. Schwendicke, C. Dorfer, S. Kneist, H. Meyer-Lueckel, S. Paris, Cariogenic effects of probiotic Lactobacillus rhamnosus GG in a dental biofilm model, Caries Res. 48 (2014) 186-192.

[33] W. Krzysciak, D. Koscielniak, M. Papiez, P. Vyhouskaya, K. Zagorska-Swiezy, I. Kolodziej, et al., Effect of a Lactobacillus salivarius probiotic on a double-species Streptococcus mutans and Candida albicans caries biofilm, Nutrients 9 (2017).

[34] A. Maldonado, J.L. Ruiz-Barba, R. Jimenez-Diaz, Purification and genetic characterization of plantaricin NC8, a novel coculture-inducible two-peptide bacteriocin from Lactobacillus plantarum NC8, Appl. Environ. Microbiol. 69 (2003) 383-389.

[35] P. Wannun, S. Piwat, R. Teanpaisan, Purification, characterization, and optimum conditions of fermencin SD11, a bacteriocin produced by human orally Lactobacillus fermentum SD11, Appl. Biochem. Biotechnol. 179 (2016) 572-582.

[36] J. Ge, Y. Sun, X. Xin, Y. Wang, W. Ping, Purification and partial characterization of a novel bacteriocin synthesized by Lactobacillus paracasei HD1-7 isolated from Chinese sauerkraut juice, Sci. Rep. 6 (2016) 19366.

[37] R. Wasfi, O.A. Abd El-Rahman, M.M. Zafer, H.M. Ashour, Probiotic Lactobacillus sp. inhibit growth, biofilm formation and gene expression of caries-inducing Streptococcus mutans, J. Cell Mol. Med. 22 (2018) 1972-1983.

[38] K.B. Ahn, J.E. Baik, O.J. Park, C.H. Yun, S.H. Han, Lactobacillus plantarum lipoteichoic acid inhibits biofilm formation of Streptococcus mutans, PLoS One 13 (2018) e0192694. 